\title{
Low-energy subgap states and the magnetic flux periodicity in $d$-wave superconducting rings
}

\author{
Yu. S. Barash \\ Institute of Solid State Physics, Russian Academy of Sciences, Chernogolovka, Moscow District, 142432 Russia
}

(Dated: September 15, 2021)

\begin{abstract}
Wave functions of low-energy quasiparticle subgap states in $d$-wave superconducting rings, threaded by Aharonov-Bohm magnetic flux, are found analytically. The respective energies are closest to the midgap position at small magnetic fluxes and deviate from the Fermi surface due to the Doppler shift, produced by the supercurrent. The Doppler-shifted zero-energy states result in a paramagnetic response of the ring at small fluxes. The states exist only for even angular momenta of the center of mass of Cooper pairs, in agreement with recent numerical studies of the problem. This macroscopic quantum effect in $d$-wave rings results in broken $h / 2 e$ periodicity, retaining only the $h / e$ periodic behavior of the supercurrent with varying magnetic flux.
\end{abstract}

PACS numbers: 74.20.Rp, 74.78.Na, 74.25.Ha

The $h / 2 e$ periodic dependence of the current on the magnetic flux in superconducting rings and hollow cylinders is considered frequently as an inevitable consequence of the electronic pairing in superconductors. This point of view is fully supported by the Ginzburg-Landau theory, which contains only the Cooper pair charge $2 e$ and always predicts the magnetic flux period $h / 2 e$. Numerous experimental results seem to be also in favor of such conclusions, including first observations of the $h / 2 e$ flux quantization in hollow cylinders 1, 2], the Little-Parks effect [3] and the flux quantization of Abrikosov vortices [4, 5]. However, the Ginzburg-Landau approach applies on the scale much greater than the Cooper pair size and, strictly speaking, at temperatures near $T_{c}$. It is, in general, not applicable to mesoscopic rings at low temperatures, where a microscopic approach, which is not so plain with respect to the problem [6, 7, 8], has to be explored.

According to the microscopic BCS theory, normalmetal electrons with projections $\hbar M$ and $\hbar \bar{M}$ of their orbital angular momenta along the ring axis, form in $s$ wave superconductors Cooper pairs with the angular momentum $\hbar q=\hbar(M+\bar{M})$ of their center of mass [6, 9 ]. Respectively, a Bogoliubov quasiparticle in the superconducting ring represents a superposition of an electron with the angular momentum $\hbar M$ and a hole with $-\hbar \bar{M}$, and the difference is $\hbar q$. Since $M$ and $\bar{M}$ have to be integers and can be represented under the conditions in question as $M=(\ell+q) / 2$ and $\bar{M}=(-\ell+q) / 2$, the quantities $\ell$ and $q$ can take simultaneously either even or odd integral values [6, [9]. The key point is that the single valuedness of the wave functions is protected by the quantities $(\ell \pm q) / 2$, whereas only $q / 2$ enters the expression for the supercurrent via a standard combination $q / 2-\Phi / \Phi_{0}$ with the magnetic flux. As a result, the factor $q-2 \Phi / \Phi_{0}$ arises in the microscopic BCS theory and leads to the magnetic flux quantum $\Phi_{0} / 2$ in superconductors, a half of the normal-state flux quantum $\Phi_{0}=h / e$ [10, 11, 12].
The condition of the single valuedness of the wave function, which justifies the presence of the superconducting flux quantum $\Phi_{0} / 2$, does not necessarily result itself in the $h / 2 e$ periodicity, however. An important additional condition, which has to be satisfied for ensuring the periodicity, is the degeneracy of the respective states. In general, the degeneracy takes place between states of a superconducting ring pierced by magnetic fluxes, which differ by an integral number of $\Phi_{0}$, i. e. by an even number of the superconducting magnetic flux quanta $\Phi_{0} / 2=h / 2 e$. A difference by odd numbers of the quanta is, in principle, physically distinguished [6, 7]. This circumstance represents a significant interest since it could lead to a $h / e$-periodic component in behaviors of superconducting rings, which removes the specific superconducting $h / 2 e$ periodicity or, at least, makes it approximate.

On the other hand, there are microscopic arguments, which substantially restrict possible deviations from the $h / 2 e$ periodicity and explain from the microscopic point of view its numerous experimental observations in superconducting rings. The arguments give also a general idea for further search for the conditions, when the breaking of the periodicity is noticeable. As this follows from the BCS theory, the breaking could become observable, if, in carrying out the statistical averaging, one cannot always replace the sum over states near the Fermi surface by the respective integral. For this reason the discreteness of the angular momentum is of crucial importance for making it possible to distinguish between the states of a ring, which differ by odd superconducting flux quanta. For $s$ wave pairing, this can happen in mesoscopic rings with characteristic sizes of the order of the coherence length $\xi_{0}$ or less [7]. Among various possible complications of the standard BCS approach which arise in rings of such a small size, probably, the main one is that the discreteness of the states and the pair breaking effect of the supercurrent can destroy the superconductivity there, at least, within some range of the magnetic flux [13]. This com- 
plicates an experimental observation of the magnetic flux periodicity in a superconducting state of the mesoscopic $s$-wave rings. The fluctuations of the type of quantum phase slips, as well as effects of the Coulomb blockade, could also play an important role there.

The problem has been investigated recently in Ref.8 8 for $d$-wave rings at low temperatures, where a noticeable violation of the $h / 2 e$ periodicity, associated with discrete current-carrying low-energy states, was identified. One of the most striking results of Ref $[8$, obtained within numerical selfconsistent calculations, is that in $d$-wave superconducting rings quasiparticle subgap states with quite low energies exist only for even $q$. By contrast, a considerable empty spectral gap appears in $d$-wave rings with odd $q$. In the presence of low-energy states, a comparatively small variation of the Doppler shift with the magnetic flux turns out to be sufficient for generating an alternation of the diamagnetic and paramagnetic states in the ground state of the $d$-wave ring. As a result, the breaking of the $h / 2 e$ periodic dependence of the supercurrent on the magnetic flux has been found to be observable in $d$-wave rings, whose size can significantly exceed the coherence length $\xi_{0}$.

In the present paper an analytical approach will be developed for studying the effect, based on a comparatively simple model of the $d$-wave superconducting ring. Energies and wave functions of quasiparticle current-carrying states, lying closest to the Fermi level at small magnetic fluxes through the rings, will be obtained by solving the respective quasiclassical equations. The states turn out to deviate from the zero energy (the midgap position) only due to the Doppler shift, produced by the supercurrent. They form a paramagnetic current, which dominates the response of the ring at low fluxes. Since the anisotropic $d$-wave pairing breaks the conservation of the angular momentum along the ring axis, the angular dependence of the respective quasiparticle wave functions does not reduce to a simple exponential dependence $\exp [i(\ell \pm q) \varphi / 2]$, which they would possess in isotropic $s-$ wave or normal-metal rings. The probability density of the states has its maxima at the nodes of the order parameter.

The phase factors of the quasiparticle wave functions are also modified in $d$-wave rings. This results in a topological reason for the low-energy quasiparticle states to survive only for even $q$. It will be shown, that the quantity $\ell / 2$ in the phase factor is replaced by the value $M_{F}$ of the angular momentum along the ring axis of the normalstate quasiparticles at the Fermi energy, for a given transverse channel. Since $2 M_{F}$ is always even, the exponential $\exp \left[i\left(2 M_{F} \pm q\right) \varphi / 2\right]$ is compatible with the single valuedness of the superconductor wave-function only for even $q$ and, hence, for even $\ell$. Therefore, the Doppler-shifted zero-energy states exist only in even $q$-sectors of the pairing. This signifies that the violation of the $h / 2 e$ periodicity in the subgap spectrum of $d$-wave superconducting rings is a macroscopic quantum effect associated with the discreteness of the states and the specific structure of their wave functions. The discreteness is of the universal character and should be observable at sufficiently low temperatures, when pairings with different $q$ in superconducting rings can be distinguished.

Subgap states Consider a narrow strongly type II superconducting ring with a negligible supercurrent-induced magnetic flux, where the arm width $L$ is much less than the penetration depth $L \ll \lambda_{L}$ and considerably exceeds the coherence length. The nonlocal operator for an anisotropic order parameter in the ring can be described quasiclassically and reduced, in the main approximation, to a local angular dependent quantity.

The dependence of the Bogoliubov amplitudes on the polar angle in continuous circular (cylindrical) rings can be taken in the form

$$
\left\{\begin{array}{l}
u(\varphi)=\tilde{u}(\varphi) \exp \left[\frac{i}{2}(\ell+q) \varphi\right] \\
v(\varphi)=\tilde{v}(\varphi) \exp \left[\frac{i}{2}(\ell-q) \varphi\right] \\
\Delta(\varphi)=\tilde{\Delta}(\varphi) \exp (i q \varphi)
\end{array}\right.
$$

Quantities $\tilde{u}$ and $\tilde{v}$ do not depend on the polar angle $\varphi$ in isotropic normal metals and $s$-wave superconductors. Since in $d$-wave rings the angular dependence of amplitudes $\tilde{u}(\varphi)$ and $\tilde{v}(\varphi)$ is induced entirely by an anisotropy of the superconducting order parameter $\tilde{\Delta}(\varphi)$ and disappears in the normal-metal state, the amplitudes vary comparatively slowly with changing $\varphi$. Taking this into account, the following equations of the Andreev type for $\tilde{u}(\varphi)$ and $\tilde{v}(\varphi)$ can be derived:

$$
\begin{aligned}
& i \frac{\hbar \mathrm{v}_{F, \varphi}}{R} \frac{d \tilde{u}}{d \varphi}+\left(\varepsilon-\xi-m \mathrm{v}_{F, \varphi} \mathrm{v}_{s}\right) \tilde{u}-\tilde{\Delta}(\varphi) \tilde{v}=0 \\
& i \frac{\hbar \mathrm{v}_{F, \varphi}}{R} \frac{d \tilde{v}}{d \varphi}-\left(\varepsilon+\xi-m \mathrm{v}_{F, \varphi} \mathrm{v}_{s}\right) \tilde{v}+\tilde{\Delta}^{*}(\varphi) \tilde{u}=0 .
\end{aligned}
$$

Here $\mathrm{v}_{s}$ is the supercurrent velocity, which takes the form $2 m R \mathrm{v}_{s}=\hbar \min _{q}\left(q-2 \Phi / \Phi_{0}\right) ; \Phi$ is the external Aharonov-Bohm magnetic flux and the associated vectorpotential is $A_{\varphi}=\Phi / 2 \pi R$. The normal-state quasiparticle excitation energy $\xi$ is taken with respect to the Fermi level. For a given transverse channel, $\xi$ is a discrete quantity, which depends on $\ell / 2$ in Eqs.(2), and the effective Fermi velocity of a circular motion is defined as $\mathrm{v}_{F, \varphi}=\partial \xi /\left.\partial p_{\varphi}\right|_{\mathbf{p}_{F}}, p_{\varphi}=\hbar \ell / 2 R$.

As the radius of the ring is much greater than the atomic scale $k_{F} R \gg 1$, the quasiclassical approach applies to a circular quasiparticle motion and, in particular, admits the condition $\left|\mathrm{v}_{F, \varphi}\right| \gg\left|\mathrm{v}_{s}\right|$, i. e. $|\ell| \gg$ $\left|q-2 \Phi / \Phi_{0}\right|$. For the maximum value of the superfluid velocity $\left|m \mathrm{v}_{F, \varphi} \mathrm{v}_{s}\right| \sim \max [|\Delta(\varphi)|]$, one gets $\left|\mathrm{v}_{s}\right| \sim$ $\left(k_{F} \xi_{0}\right)^{-1}\left|\mathrm{v}_{F, \varphi}\right| \ll\left|\mathrm{v}_{F, \varphi}\right|$. Hence, linear terms in $\mathrm{v}_{s}$, as well as all other terms in Eqs.(2), can be considered 
within the quasiclassical description as small quantities of the first order as compared with $\varepsilon_{F} \tilde{u}(\varphi)$.

In $s$-wave superconductors, the amplitudes $\tilde{u}, \tilde{v}$ do not depend on $\varphi$ and the condition for nontrivial solutions of Eqs. (2) results in the quasiparticle energies

$$
\varepsilon=m \mathrm{v}_{F, \varphi} \mathrm{v}_{s} \pm \sqrt{\xi^{2}+|\Delta|^{2}}
$$

The ground-state value of $q$ is known to possess the $h / 2 e$ period, changing with flux as $q=\operatorname{int}\left[2 \Phi / \Phi_{0}+\right.$ $\operatorname{sgn}(\Phi) / 2$ ], up to small finite-size corrections. Such a dependence is assumed observable below, for example, if the system is field cooled through $T_{c}$ for each field value. In general, states with different $q$ describe different metastable phases of the superconductor [9], which usually possess large lifetimes, even if $L \ll \lambda_{L}$ and the magnetic flux is not really trapped. If pair breaking effects of the supercurrent are small, the energies (31) depend linearly on $q-2 \Phi / \Phi_{0}$ and manifest the same $h / 2 e$ periodic dependence.

In the absence of the supercurrent, energies (31) coincide with those in homogeneous superconductors. The Doppler shift, described by the first term in Eq.(3), causes some of the states to move in the subgap region with varying magnetic flux. As $\left[q-(2 \Phi)\left(\Phi_{0}\right)\right] \leq 1 / 2$ for the ground-state behavior of $q$, the energy (3) can cross the Fermi surface at some flux value only in small rings, when $4 R<\hbar \mathrm{v}_{F, \varphi} /|\Delta|$ and the pair breaking supercurrent strongly modifies or even destroys the superconducting state in the ring. In $s$-wave rings of larger size, energies of the subgap states lie comparatively far from the midgap position.

Andreev equations (2) can be transformed to Riccati equation [14], which has periodic coefficients in a doubly connected $d$-wave sample. Numerous solutions of Eqs. (2) will not be discussed analytically here, as this is mostly the problem for numerical studies. The selfconsistent numerical solution of the respective Bogoliubov-de Gennes equations for the tight-binding model of the dwave square loops is represented in Ref 8 . There are, however, two remarkably simple degenerate continuous solutions of Eqs.(2) for an intrinsically real order parameter $\tilde{\Delta}(\varphi)$, which changes its sign and vanishes after averaging over the polar angle. These solutions are of special interest and can be described analytically for any particular angular dependence $\tilde{\Delta}(\varphi)$. For a simple model of the $d_{x^{2}-y^{2}}$-wave order parameter, one can write in the main quasiclassical approximation $\tilde{\Delta}(\varphi)=\Delta_{d} \cos 2 \varphi$ after applying $\Delta$ to (1). For spatially constant $\Delta_{d}$, the "electron-like" and the "hole-like" solutions take the following form (in a more general case one should make the

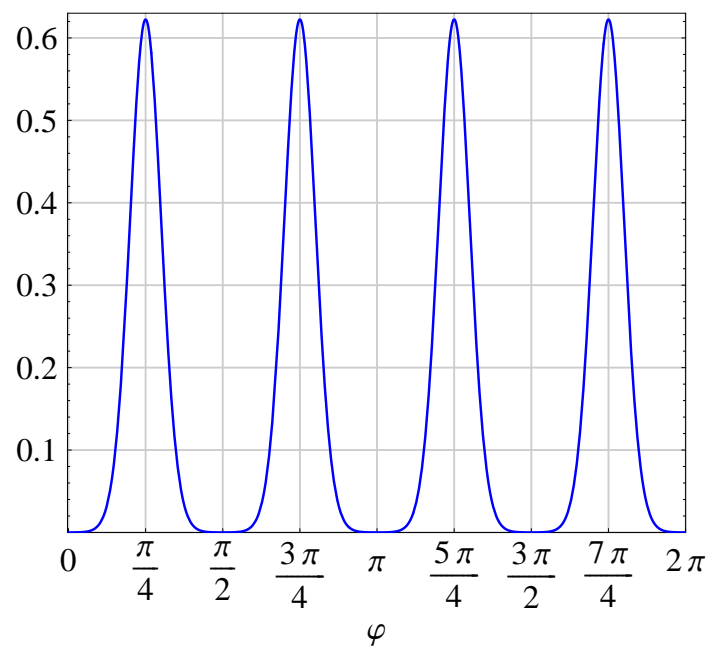

FIG. 1: The probability density as a function on the polar angle for the Doppler-shifted zero-energy state in the d-wave ring with $R=10 \hbar \mathrm{v}_{F, \varphi} /\left|\Delta_{d}\right|$.

substitution in Eqs. (4), (5) $\left.\Delta_{d} \sin (2 \varphi) / 2 \rightarrow \int \tilde{\Delta}(\varphi) d \varphi\right)$ :

$$
\begin{aligned}
& \left(\begin{array}{l}
\tilde{u}(\varphi) \\
\tilde{v}(\varphi)
\end{array}\right)=C_{1} \exp (-i \beta \varphi)\left(\begin{array}{c}
\cosh \left(\frac{\Delta_{d} R}{2 \hbar \mathrm{v}_{F, \varphi}} \sin 2 \varphi\right) \\
i \sinh \left(\frac{\Delta_{d} R}{2 \hbar \mathrm{v}_{F, \varphi}} \sin 2 \varphi\right)
\end{array}\right), \\
& \left(\begin{array}{l}
\tilde{u}(\varphi) \\
\tilde{v}(\varphi)
\end{array}\right)=C_{2} \exp (-i \beta \varphi)\left(\begin{array}{l}
\sinh \left(\frac{\Delta_{d} R}{2 \hbar \mathrm{v}_{F, \varphi}} \sin 2 \varphi\right) \\
i \cosh \left(\frac{\Delta_{d} R}{2 \hbar \mathrm{v}_{F, \varphi}} \sin 2 \varphi\right)
\end{array}\right),
\end{aligned}
$$

where $\beta=R \xi / \hbar \mathrm{v}_{F, \varphi}, \varepsilon=m \mathrm{v}_{F, \varphi} \mathrm{v}_{s}$ and constant amplitudes $C_{1,2}$ result from the normalization.

The energy $\varepsilon= \pm m\left|\mathrm{v}_{F, \varphi} \mathrm{v}_{s}\right|$ deviates from the midgap position due to the Doppler shift and its sign depends on relative directions of the quasiparticle and the supercurrent circulations. If the ring size noticeably exceeds $\xi_{0}$, then $|\varepsilon| \ll\left|\Delta_{d}\right|$. The origin of the Doppler-shifted zeroenergy states is associated with the change of sign of the $d$-wave order parameter. Fig. [1 displays the probability density of the states, which possesses the characteristic fourfold structure and reaches its maximum exactly at the nodes $\varphi= \pm \frac{\pi}{4}, \pm \frac{3 \pi}{4}$. As for superpositions of (4) and (5) the fourfold symmetry reduces to two twofold structures, the degeneracy of the states (4) and (5) could be lifted within higher-order approximations.

The applicability of the quasiclassical approach to the solutions (4) and (5) can be explicitly justified. The terms containing the derivatives of the first order in Eqs. (2), can be estimated as $\left(\hbar \mathrm{v}_{F, \varphi} / R\right) d \tilde{u}(\varphi) / d \varphi \sim \Delta_{0} \tilde{u}(\varphi)$, or $\sim \xi \tilde{u}(\varphi)$, and identified as small quantities of the first order. At the same time, the terms which have been 
neglected within the accuracy of the Eqs.(2), contain an additional small factor $\Delta_{0} / \varepsilon_{F}$ or $\xi / \varepsilon_{F}$ as compared to the first-order terms. This concerns, in particular, the terms with the second-order derivatives, which can be, therefore, classified as small quantities of the second-order. A variation of the coefficient $\hbar \mathrm{v}_{F, \varphi} / R$ in front of the firstorder derivatives in Eqs.(2), associated with the difference between $\mathrm{v}_{\varphi}$ and the respective polar component of the Fermi velocity $\mathrm{v}_{F, \varphi}$, also results in small terms of the second order, which are beyond the accuracy of the equations and have to be neglected. This circumstance is important for further considerations.

Broken h/2e periodicity Wave functions of the orbital motion have to be single-valued, if only single-valued gauge transformations are used and spin is not intimately involved in the problem [15, 16]. This is the case, in particular, for the Bogoliubov amplitudes (1), (4) and (51). The exponentials in Eq.(1) are always single-valued, since $\ell$ and $q$ take even or odd values only simultaneously. Hence, the amplitudes $\tilde{u}(\varphi)$ and $\tilde{v}(\varphi)$ have to be singlevalued themselves and their phases have to acquire an integer number of $2 \pi$ after going around the loop. This signifies that the solutions (44), (5) really exist only if $\beta$ is an integer. For the last condition to be satisfied, the quantization of the angular momentum along the ring axis turns out to be of crucial importance. Indeed, close to the Fermi surface $\left|(\ell / 2)-M_{F}\right| \ll\left|M_{F}\right|$ and the quantized normal-state excitation energy $\xi(\ell / 2)$ takes the following form: $\xi(\ell / 2)=\mathrm{v}_{F, \varphi}\left(p_{\varphi}-p_{F, \varphi}\right)=\frac{\hbar \mathrm{v}_{F, \varphi}}{R}\left(\ell / 2-M_{F}\right)$. Therefore, $\beta(\ell / 2)=\left(\ell / 2-M_{F}\right)$. As $M_{F}$ is always an integer, $\beta$ is an integer-valued parameter for even $\ell$ and, hence, for even $q$. In the case of odd $\ell$ and $q$ the parameter $\beta$ takes half-integer values, however. For this reason the Bogoliubov amplitudes (11), (4) and (5) are not singlevalued for odd $q$, changing their sign after going around the loop. Hence, the Doppler-shifted zero-energy states (44), (5) exist in the $d$-wave superconducting rings in even $q$-sectors of the pairing, whereas for odd $q$ the states do not arise in the gap.

As a rule, $\xi$ can be effectively excluded from a theoretical analysis of observable superconductor properties. For example, the current and other observables are expressed via quasiclassical Green functions, which are $\xi$ integrated. As this follows from the present paper, the situation can change substantially, if $\xi$ takes discrete values due to the angular momentum quantization.

Paramagnetic response The total current $J$ induced by the Aharonov-Bohm field $A_{\varphi}(\rho)=\Phi / 2 \pi \rho$ in a cylindrical ring, is obtained from the relation $(\delta \mathcal{E}(\Phi))_{S}=$ $-(1 / c) \int_{V} j_{\varphi}(\rho) \delta A_{\varphi}(\rho) d V=-(1 / c) J \delta \Phi$, where $\mathcal{E}$ is the energy and $S$ the entropy. Thus, in even $q$-sectors of the pairing, a contribution to the subgap current from the two states with $\pm \ell / 2$ takes the form $J=$ $-\left(e \mathrm{v}_{F, \varphi} / 2 \pi R\right) \tanh \left[\left(\hbar \mathrm{v}_{F, \varphi} / 4 R T\right)\left(q-\frac{2 \Phi}{\Phi_{0}}\right)\right]$. In taking the derivative of the energy over the magnetic flux, abrupt changes associated with the $\delta q= \pm 1$ transitions have been disregarded. Within the model, the characteristic value of the current $e \mathrm{v}_{F, \varphi} / 2 \pi R$, carried by the lowenergy states, coincides with that for the normal-state persistent current [17]. However, its sign is determined by the quantity $q-\frac{2 \Phi}{\Phi_{0}}$, which is specific for the superconducting state. As a result, it is a paramagnetic current. The current dominates the magnetic response of the ring in vicinities of centers of even $q$-sectors, including small fluxes at $q=0$, where energies of the states (4), (5) are most close to the Fermi surface. The paramagnetic response of the zero-energy states is known to take place also near surfaces of $d$-wave superconductors [18, 19].

Conclusion Energies and wave functions of the lowenergy quasiparticle states have been obtained analytically in $d$-wave superconducting rings threaded by an Aharonov-Bohm magnetic flux. The states turn out to deviate from the zero energy due to the supercurrentinduced Doppler shift. They form a paramagnetic response of the rings at small fluxes. The Doppler-shifted zero-energy states are found to exist only for pairings with even angular momenta of the center of mass of Cooper pairs. This macroscopic quantum effect breaks $h / 2 e$ periodic behavior of the supercurrent in the ring, in agreement with the results of Ref:8. The analytical approach developed in the present work, demonstrates explicitly that the quantization of the orbital angular momentum and the condition for the single valuedness of the superconductor wave function play the key role in a formation of the difference between quasiparticle subgap spectra at even and odd $q$.

Acknowledgements I am grateful to F. Lodder, A. Kampf, T. Kopp and J. Mannhart for useful discussions. The support of RFBR grant 08-02-00842 is acknowledged.

[1] R. Doll and M. Näbauer, Phys. Rev. Lett. 7, 51 (1961).

[2] B. S. Deaver and W. M. Fairbanks, Phys. Rev. Lett. 7, 43 (1961).

[3] R. D. Parks and W. A. Little, Phys. Rev. Lett. 9, 9 (1962).

[4] A. A. Abrikosov, Soviet Physics - JETP 5, 1174 (1957).

[5] U. Essmann and H. Träuble, Phys. Lett. A 24, 526 (1967).

[6] J. R. Schrieffer, Theory of Superconductivity, Addison Wesley Publishing Co, Inc. (1964).

[7] P. G. de Gennes, Superconductivity of Metals and Alloys, Addison Wesley Publishing Co, Inc. (1966).

[8] F. Loder, A. P. Kampf, T. Kopp, J. Mannhart, C. W. Schneider, and Y. S. Barash, Nature Physics 4, 112 (2008).

[9] A. Bohr, B. R. Mottelson, Phys. Rev. 125, 495 (1962).

[10] N. Byers and C. N. Yang, Phys. Rev. Lett. 7, 46 (1961).

[11] L. Onsager, Phys. Rev. Lett. 7, 50 (1961).

[12] W. Brenig, Phys. Rev. Lett. 7, 337 (1961). 
[13] K. Czajka, M. Maśka, M. Mierzejewski, and Z. Śledź, Phys. Rev. B 72, 035320 (2005).

[14] Y. Nagato and K. Nagai, J. Low Temp. Phys. 93, 33 (1993).

[15] E. Merzbacher, Am. J. Phys. 30, 237 (1962).

[16] S. Olariu, I. I. Popescu, Rev. Mod. Phys. 57, 339 (1985).

[17] Y. Ymry, Introduction to mesoscopic physics, Oxford
University Press, New York, 2002.

[18] Yu.S. Barash, M.S. Kalenkov, J. Kurkijärvi, Phys. Rev. B 626665 (2000).

[19] A. Carrington, F. Manzano, R. Prozorov, R.W. Giannetta, N. Kameda, and T. Tamegai, Phys. Rev. Lett. 86, 1074 (2001). 